\title{
Short-term Reliability Model Based on The Probability Distribution of Power Transmission and Transformation Equipment
}

\author{
Chao Huang ${ }^{1, a}$, Xiaoquan $\mathrm{Li}^{1, \mathrm{~b}}$, Shuai Liu ${ }^{1, \mathrm{C}}$ \\ ${ }^{1}$ Air force Engineering University, College of Air and Missile Defense, Xi'an Shaan Xi, \\ 710051,China; \\ a379068078@qq.com,, ${ }^{\mathrm{b}}$ LXQ@126.com, ${ }^{\mathrm{C}}$ masteryliu@163.com
}

Keyword: Power transmission equipment, Opreation reliability, Probability model, Weibull distribution

\begin{abstract}
For power transmission equipment long-term planning in the steady-state failure isn't suitable for using to predict the reliability of the short-term problems, according to the result in transmission equipment shut down three different reasons: overload protection action, the weather factor, aging failure, choose normal distribution, index distribution, based on the minimum estimation and average rank method of weibull distribution to establish the corresponding reliability probability model of transmission equipment. Examples are calculated by transformer shut down probability under different condition, results show that the model can correctly reflect the practical operation of transformer in different situations. The model can predict the failure rate of the power transmission equipment in the short term.
\end{abstract}

\section{Introduction}

Found after summarizing the research achievements of predecessors, to predict the short-term reliability of power transmission and transformation equipment level almost adopt constant equipment failure rate of the long-term planning of power system equipment failure ${ }^{[1]}$,the constant equipment failure rate is not fit to used for power transmission and transformation equipment operation reliability modeling.

In this paper, according to different power transmission and transformation equipment stoppage reasons, the failure model of power transmission and transformation equipment short-term operation can be divided into three categories: overload protection failure model, weather failure model and aging failure model, Aimed at the three different kinds of failure model, respectively choose normal distribution, exponential distribution, and the average rank estimation based on the least square method of weibull distribution to establish the model for failure probability. This model can predict short-term fault level of power transmission and transformation equipment and be used in the short-term operation of the power transmission and transformation equipment risk assessment.

\section{Based on the normal distribution of power transmission and transformation equipment overload protection failure model}

Power transmission and transformation equipment both have the shut down situation because too much electricity lead to overload protection system action, overload protection system mainly consists of relay protection and mutual inductor protection, but both overload protection device current trigger value has certain top and bottom line error, need to take into account in the calculation of the error value ${ }^{[2]}$.

According to the above analysis, and set the overload protection system to trigger current value is $I_{\text {limit }}$,up and down the line for the error is $\lambda, I_{\text {limit }}$ obey the average $I_{\text {limito }}$,standard deviation $\sigma$,range for $\left[I_{\text {limit } 0}(1-\lambda), I_{\text {limit } 0}(1+\lambda)\right]$ normal distribution, the density function $I_{\text {limit }}$ can be expressed as: 


$$
f\left(I_{\text {limit }}\right)=\left\{\begin{array}{cc}
\frac{1}{t \sigma \sqrt{2 \pi}} \exp \left[-\frac{\left(I_{\text {limit }}-I_{\text {limit }}\right)^{2}}{2 \sigma^{2}}\right], & I_{\text {limit }} \in\left[I_{\text {limito }}(1-\lambda), I_{\text {limito }}(1+\lambda)\right] \\
0, & I_{\text {limit }} \notin\left[I_{\text {limit }}(1-\lambda), I_{\text {limito }}(1+\lambda)\right]
\end{array}\right.
$$

Among them:

$$
t=\Phi\left(\frac{I_{\text {limit } 0} \lambda}{\sigma}\right)-\Phi\left(\frac{-I_{\text {limito }} \lambda}{\sigma}\right)
$$

$\Phi$ is the standard normal distribution function.

Overload protection misoperation protection situation, when the current value is less than $I_{\text {limit }}$,overload protection device working cause components to halt, so you need to consider the probability that the load protection misoperation.

For example, power lines overload protection failure probability model is derived. Set the transmission line actual load current as $I, P_{l a}$ is the total overload protection action outage rate, $P_{l n}$ is the probability of correct action for load protection system, $P_{l u}$ is the probability of misoperation is the overload protection system, $A_{1}$ is $I \geq I_{\text {limit }}, A_{2}$ is $I \leq I_{\text {limit }}$, according to the definition of full probability formula available:

$$
P_{l a}(I)=P_{l n} P\left(A_{1}\right)+P_{l u} P\left(A_{2}\right)
$$

$P_{l n}$ 和 $P_{l u}$ can be obtained according to the historical statistical data analysis, classification after discussion:

When $I<I_{\text {limito }}(1-\lambda), \quad P\left(A_{1}\right)=0, \quad P\left(A_{2}\right)=1$, there are:

$$
P_{l a}(I)=P_{l u}
$$

When $I_{\text {limit } 0}(1-\lambda) \leq I \leq I_{\text {limito }}(1+\lambda)$, there are:

$$
P_{l a}(I)=P_{l n} \int_{I_{\text {limitio }}(1-\lambda)}^{I} f\left(I_{\text {limit }}\right) \mathrm{d} I_{\text {limit }}+P_{l u} \int_{I}^{I_{\text {limito }}(1+\lambda)} f\left(I_{\text {limit }}\right) \mathrm{d} I_{\text {limit }}
$$

When $I>I_{\text {limit } 0}(1+\lambda), \quad P\left(A_{1}\right)=1, \quad P\left(A_{2}\right)=0$, there are:

$$
P_{l a}(I)=P_{l n}
$$

Overload protection failure model of the transmission line is deduced from the above analysis, due to the size of the load level reference of the transfomer is also the current, so transformer overload protection action to process reference transmission lines can be derived from the transmission lines model.

\section{Based on the exponential distribution of power transmission and transformation equipment weather failure model}

Power transmission and transformation equipment outside the actual operation process, encounter blizzard, the failure rate of hurricanes and other extreme weather will rise sharply. Aiming at this situation, this article could be divided into normal weather, bad weather and extreme three kinds of basic situation, under the basic conditions of three kinds of research power system power transmission and transformation equipment weather factors failure model.

In all of the statistical time, the duration of normal weather, bad weather and the extreme weather are respectively $N_{1}, N_{2}, N_{3}$; Transmission components in normal weather, bad weather, extreme weather the failure rate of $\lambda_{1}, \lambda_{2}, \lambda_{3}$; Bad weather, extreme weather failure of total failure ratio is $C_{1}, C_{2}$; Statistical period, all power transmission and transformation equipment failure rate of the statistical average $\bar{\lambda}$ can be obtained:

$$
\bar{\lambda}=\frac{N_{1}}{N_{1}+N_{2}+N_{3}} \lambda_{1}+\frac{N_{2}}{N_{1}+N_{2}+N_{3}} \lambda_{2}+\frac{N_{3}}{N_{1}+N_{2}+N_{3}} \lambda_{3}
$$


The relationship $C_{1}, C_{2}$ between and failure rate $\lambda_{2}, \lambda_{3}$ is:

$$
\begin{aligned}
& C_{1}=\frac{N_{2} \lambda_{2}}{\left(N_{1}+N_{2}+N_{3}\right) \bar{\lambda}} \\
& C_{2}=\frac{N_{3} \lambda_{3}}{\left(N_{1}+N_{2}+N_{3}\right) \bar{\lambda}}
\end{aligned}
$$

Vertical7 9, the failure rate $\lambda_{1}, \lambda_{2}, \lambda_{3}$ can be expressed:

$$
\begin{aligned}
& \lambda_{1}=\frac{N_{1}+N_{2}+N_{3}}{N_{1}} \bar{\lambda}\left(1-C_{1}-C_{2}\right) \\
& \lambda_{2}=\frac{N_{1}+N_{2}+N_{3}}{N_{1}} \bar{\lambda} C_{1} \\
& \lambda_{3}=\frac{N_{1}+N_{2}+N_{3}}{N_{1}} \bar{\lambda} C_{2}
\end{aligned}
$$

To introduce the weather type indicator variable $w_{i}$, said power transmission and transformation equipment in current weather conditions, $i=1,2,3$ represent the normal weather, bad weather, extreme weather. Formula 10 12 can be represented as:

$$
\lambda\left(w_{i}\right)=\left\{\begin{array}{c}
\lambda_{1}=\frac{N_{1}+N_{2}+N_{3}}{N_{1}} \bar{\lambda}\left(1-C_{1}-C_{2}\right) w_{i}=1 \\
\lambda_{2}=\frac{N_{1}+N_{2}+N_{3}}{N_{1}} \bar{\lambda} C_{1} \quad w_{i}=2 \\
\lambda_{3}=\frac{N_{1}+N_{2}+N_{3}}{N_{1}} \bar{\lambda} C_{2} \quad w_{i}=3
\end{array}\right.
$$

Statistical weather this time is kept constant, power transmission and transformation equipment failure will not change, can think time obey exponential distribution, the probability $P_{w}$ of power transmission and transformation equipment failure rate can be represented as:

$$
P_{w}=1-e^{-\lambda\left(w_{i}\right) t}
$$

The weather factor failure probability model general in the transformer and transmission line, but considering the long distance transmission line equipment may span the area of a number of different weather conditions, introduce the total failure rate of transmission line $\lambda^{\prime}$ :

$$
\lambda^{\prime}=\sum_{i=1}^{K} \lambda\left(w_{i}\right) l_{i}
$$

$\lambda\left(w_{i}\right)$ at this time on behalf of the unit length of transmission line fault rate, $l_{i}$ as the length of transmission line, long distance transmission line elements in weather factors failure probability of future time $t$ time as follows:

$$
P_{l w}=1-e^{-\lambda^{\prime} t}
$$

\subsection{Weibull distribution model and its parameter estimation method}

Double parameters of weibull distribution ${ }^{[4]}$ for the cumulative probability distribution function, reliability function, distribution density function, failure rate function respectively are:

$$
\begin{aligned}
& F(t)=1-\exp \left[-\left(\frac{t}{\eta}\right)^{m}\right] \\
& R(t)=1-F(t)=\exp \left[-\left(\frac{t}{\eta}\right)^{m}\right] \\
& f(t)=\frac{m t^{m-1}}{\eta^{m}} \exp \left[-\left(\frac{t}{\eta}\right)^{m}\right] \\
& \lambda(t)=\frac{m t^{m-1}}{\eta^{m}}
\end{aligned}
$$


Formula, $t$ time, $\eta$ is called the scale parameter or calibration parameters, $m$ called shape parameters.

The parameters of the weibull distribution model $m$ and $\eta$ are chosen to estimate the average rank method and the least-square method, On 17 take two logarithmic formula, available

$\ln \{-\ln [1-F(t)]\}=m \ln t-m \ln \eta$

make

$y=\ln \{-\ln [1-F(t)]\}, x=\ln t, a=-m \ln \eta, b=m$

have

$y=a+b x$

Want to fit parameters $a$ and $b$,you must know the value of the $F(t)$ weibull cumulative probability distribution function and fault time $t, t$ through history check fault time, $F(t)$ the average rank method for solving, average rank method can effectively improve the accuracy of the data, the average rank method to solve the formula is:

$$
\begin{aligned}
& \Delta A_{i}=\frac{n+1-A_{i-1}}{n-i+2} \\
& A_{i}=A_{i-1}+\Delta A_{i} \\
& F\left(t_{i}\right)=\frac{A_{i}-0.3}{n+0.4}
\end{aligned}
$$

Get the cumulative probability distribution function $F(t)$ and the corresponding fault time, using the least squares fitting method for linear parameter estimation method of drawing, get the regression equation of the weibull distribution, to get the shape parameters $m$ and the calibration parameters $\eta$, the resulting parameter estimates more accurate than the direct use of experience.

\subsection{Based on the Weibull distribution of power transmission and transformation equipment ageing failure model}

At present most of the transformers in the power system are oil-immersed transformers. In addition to the following transformer unless instructions are for oil-immersed transformer.

The main failure cause of ageing of the transformer is thought to be insulation failure ${ }^{[4]}$,it is generally believed is the hot spot temperature transformer decided the crucial parameters of transformer insulation life, hot spot temperature $\theta_{h s t}$ can be calculated by the IEEE guide model $^{[5]}$,according to the transformer insulation life $T_{\text {tf }}$ and the hot point temperature $\theta_{\text {hst }}$ obey Arrhenius relationship principle (Arrhenius) ${ }^{[6]}$ can get the relation between the two:

$T_{t f}=E \exp \left[\frac{15000}{\theta_{h s t}+273}\right]$

The experience of the transformer constant parameter $E$ is different, remains constant at different temperatures, the $T_{t f}=\eta$, and the formula of 27 into formulas 20 and 19 can get transformer Arrhenius Weibull model, failure rate and cumulative probability distribution function are:

$$
\begin{aligned}
& \lambda_{t}\left(t \mid \theta_{h s t}\right)=\frac{m t^{m-1}}{\left[E \exp \left(\frac{15000}{\theta_{h s t}+273}\right)\right]^{m}} \\
& F_{t}\left(t \mid \theta_{h s t}\right)=1-\exp \left[-\left(\frac{t}{E \exp \left(15000 /\left(\theta_{h s t}+273\right)\right)}\right)^{m}\right]
\end{aligned}
$$

The parameter $m$ and $E$ could be through the parameter estimation process obtained in section 3.1.

Transformer at run time $T$, in the subsequent time $\Delta t$ in ageing failure probability to shut down as follows: 


$$
P_{t c}=P\left(T \leq t \leq T+\Delta t \mid t>T, \theta_{h s t}\right)=\frac{F_{t}\left(T+\Delta t, \theta_{h s t}\right)-F_{t}\left(T, \theta_{h s t}\right)}{1-F_{t}\left(T, \theta_{h s t}\right)}
$$

Bringing formula 28 to 29 formula available transformer aging failure to shut down the probability of:

$$
P_{t c}=1-\exp \left[\left(\frac{T}{E \exp \left(15000 /\left(\theta_{h s t}+273\right)\right)}\right)^{m}-\left(\frac{T+\Delta t}{E \exp \left(15000 /\left(\theta_{h s t}+273\right)\right)}\right)^{m}\right]
$$

Aging failure model derivation of transmission lines and transformers are similar. Refer to Morgan tensile strength calculation formula of transmission line ${ }^{[6]}$, we can get power lines aging failure probability. Limited to paper length, here is no longer compose.

\section{Reliability of power transmission and transformation equipment operation total probability model}

For power transmission and transformation equipment, three failure model are independent of each other transformer, for example, transformer reliability total probability can press type calculation:

$P_{t}=P_{t a}+P_{t w}+P_{t c}-P_{t a} P_{t w}-P_{t w} P_{t c}-P_{t c} P_{t a}+P_{t a} P_{t w} P_{t c}$

$P_{t a} 、 P_{t w} 、 P_{t c}$ respectively for transformer overload protection action failure probability, weather factors failure probability, aging failure probability. The formula of transmission line refers to the transformers.

\section{The example analysis}

Transformer and transmission line the probability distribution of the same short-term reliability model application, principle, limited to space, this paper present the reliable operation of the transformer model example analysis results.

Transformer model selects the rated capacity of 800 kva medium oil circulating air-cooled transformer, transformer reliability model parameters as shown in table 1.

Table 1 Transformer reliability model parameters

\begin{tabular}{cccccc}
\hline $\begin{array}{c}\text { overload protection } \\
\text { failure model }\end{array}$ & \multicolumn{2}{c}{$\begin{array}{c}\text { weather failure } \\
\text { model }\end{array}$} & \multicolumn{2}{c}{ aging failure model } \\
\hline parameter & value & parameter & value & parameter & Value \\
$I_{\text {limit0 }}$ & 1.5 & $N_{1} / \mathrm{h}$ & 150 & & \\
$\lambda / \%$ & 9 & $N_{2} / \mathrm{h}$ & 1.5 & $m$ & 5.83 \\
$\sigma$ & 0.045 & $N_{3} / \mathrm{h}$ & 0.07 & & \\
$P_{l u}$ & 0 & $\bar{\lambda} /$ year & 0.013 & & \\
$P_{l n}$ & 1 & $C_{1}$ & 0.27 & $E$ & $1.944 * 10^{-12}$ \\
& & $C_{2}$ & 0.39 & & \\
\hline
\end{tabular}

Calculation of transformer in equivalent work for 5 years and 15 years after the first day of the service probability of every hour, transformer in the days of load current and the change of environmental temperature is shown in figure 1. 


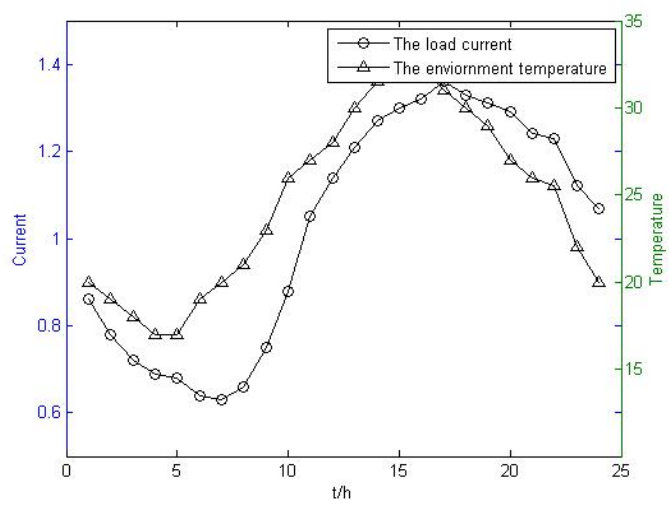

Fig.1 Transformer temperature curve and load current changes

Figure 2 for the transformer in normal weather conditions equivalent work five years later the failure probability calculation results, figure 3-5 respectively transformer equivalent work in 15 years in normal weather, bad weather and extreme weather conditions the failure probability calculation results.

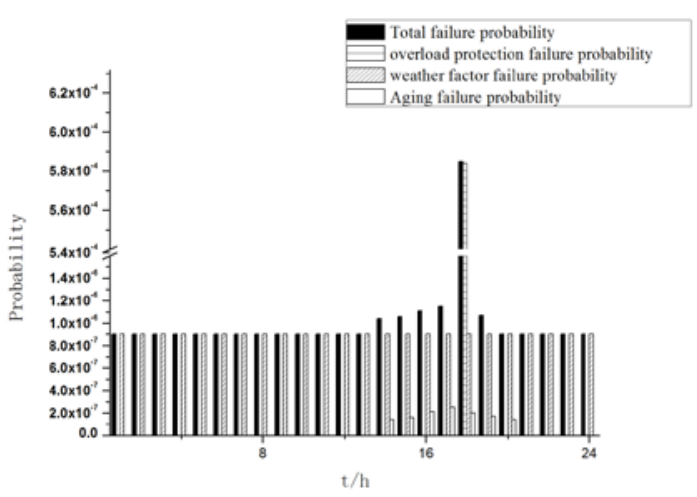

Fig 2.

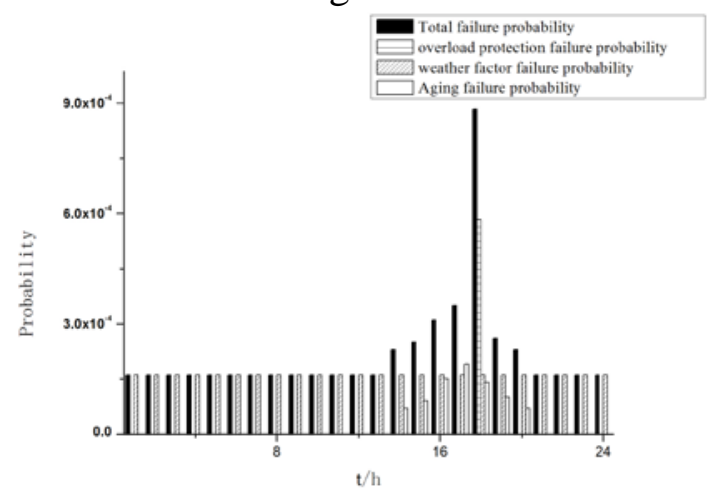

Fig 4

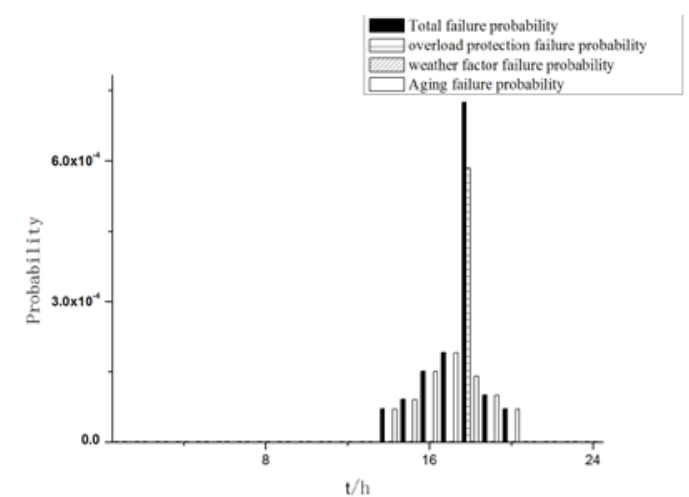

Fig3

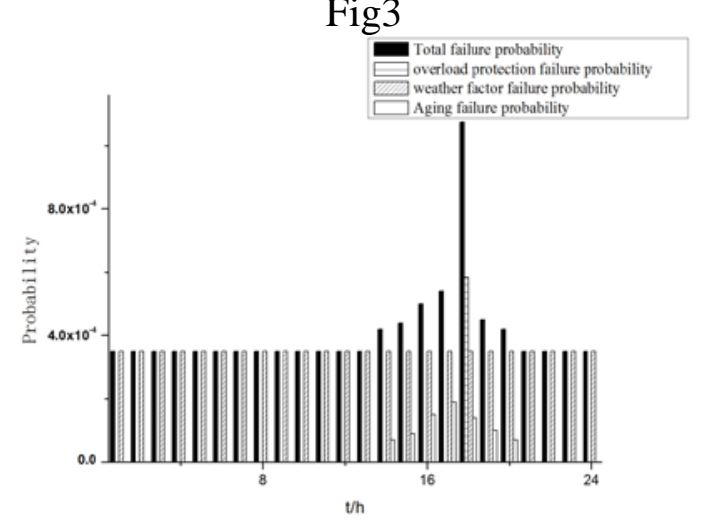

Fig5

The analysis results, available:

(1) Comparative figures 2 and 3 of the calculation result shows that when the transformer in service time is short, the increase of load current transformer will cause equipment overload protection action, power transmission and transformation equipment failure probability increases accordingly.

(2) Contrast figure 3,figure 4,figure 5 the calculation result shows that the transformer in three kinds of failure rate difference under different weather conditions, minimum under normal weather, bad weather situation next time, jump in extreme weather conditions.

(3) Compare figures 2 and 3 of the calculation result shows that in serving in the transformer fault phase with age, aging failure is the main failure forms of transformer, transformer running total failure probability with obviously increased with the increase of aging failure probability.

(4) Transformer load can be controlled by dispatching personnel, scheduling staff can lower the 
transformer load and improve the overall reliability of the transformer.

\section{Summary}

This article through to in the operation of the power transmission and transformation equipment, classify the cause of the shutdown, respectively, to choose the appropriate probability distribution established short-term reliability model of power transmission and transformation equipment, the effectiveness of the model is verified by an example. The model can predict the changes of failure rate of power transmission and transformation equipment in the short term, according to the change of the failure rate can be speculated that the reliability of the power transmission and transformation equipment in the short term.

\section{References}

[1] Billinton R, Allan R N. Reliability evaluation of power systems [M]. New York and London: Plenum Press, 1996: 182-301.

[2] Yuan Ji repair, zhuo leyou, happy, and so on. DL/T 866-2004 current transformer and voltage transformer selection and calculation guide [S]. Beijing: China power press, 2004.

[3] $\mathrm{Xu}$ Wei, wei-ming hu.Based on the two parameter weibull distribution equipment reliability prediction research. Journal of China construction machinery, 2013, 11 (2) : 15-20

[4] Chen Weigen etc. Based on the top oil temperature of transformer winding hot spot temperature calculation model improvement. Journal of chongqing university. 2012, 35 (5) : 24-29

[5] IEEE Working Group for Loading Mineral-Oil-Immersed Transformers. IEEE Std C57.91-1995 IEEE guide for loading mineral-oil-immersed transformers.

[6] Morgan V T. Effect of elevated temperature operation on the tensile strength of overhead conductor[J]. IEEE Trans. on Power Delivery, 1996, 11(1): 345-3 